\title{
Epidemiologic and histologic characteristics of CNS lesions: a 20-year experience of a tertiary center in Lebanon
}

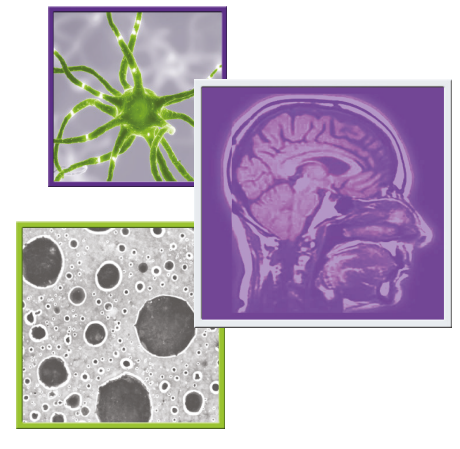

Roland Eid ${ }^{1}$, Stephanie Hage ${ }^{1}$, Ingrid Antonios ${ }^{1}$, Rita Moussa ${ }^{1}$, Makram Khoury*,1, Fady Ghassan Haddad ${ }^{1}$, Hampig Raphael Kourie ${ }^{1}$, Carole Kesrouani ${ }^{2}$, Claude Ghorra², Gerard Abadjian $^{2}$ \& Joseph Kattan ${ }^{1}$

${ }^{1}$ Department of Hematology-Oncology, Faculty of Medicine, Saint Joseph University, Beirut, Lebanon

${ }^{2}$ Department of Pathology, Faculty of Medicine, Saint Joseph University, Beirut, Lebanon

*Authors for correspondence: makram.m.khoury@gmail.com

\begin{abstract}
Aim: Report the epidemiologic and histologic characteristics of CNS lesions in the Lebanese population. Methods: We conducted a retrospective study evaluating 2025 CNS lesions diagnosed between 1998 and 2017 in the pathology laboratory of a Lebanese tertiary center. Results: $52.2 \%$ of patients were men with a median age of 50 years. The most frequent symptoms were epilepsy $(22.5 \%)$, headache $(20.6 \%)$ and motor impairment (19.9\%). 90.7\% of tumors were primary. Lung (35.6\%) and breast $(16.5 \%)$ were the most frequent primaries of metastases. $46.2 \%$ of primary CNS tumors were glial, predominantly astrocytic $(56.4 \%)$, and $(42.5 \%)$ were nonglial, predominantly meningeal tumors $(58 \%)$. Conclusion: Compared with Western literature, the Lebanese population is characterized by a younger age of onset of brain tumors, a lower rate of meningiomas and a higher rate of gliomas.
\end{abstract}

First draft submitted: 6 February 2020; Accepted for publication: 23 April 2020; Published online: 30 June 2020

Keywords: brain tumors • CNS • epidemiology • Lebanese population

The CNS is home to multiple lesions constituting complex and heterogeneous clinical and pathological different entities. These lesions can be qualified as tumoral or nontumoral, and the tumoral lesions can be benign or malignant, primary or metastatic.

More than 100 histological types of CNS tumors have been identified, thus representing a broad spectrum of clinical manifestations, treatment options and prognoses [1-3].

The CNS tumors are certainly an important cause of morbidity and mortality in children and adults, and a possible cause of significant disabilities and handicaps, and produce high burden in the patients' families and the healthcare systems [4-7].

The literature is poor in studies published on the incidence and prevalence of CNS tumors across the globe, and their epidemiology is barely understood [8,9]. In fact, only one systematic review on the worldwide incidence of brain tumors was published in 1998, subsequently another study was published in 2015 concluding that the worldwide incidence of all brain tumors is 10.82 per 100,000 person-years [8]. Although the age-adjusted annual incidence of CNS tumors is reportedly low, it is widely variable with nearly a fivefold difference between highest rates reported mainly in developed countries in the West (North America and Western Europe) and the lowest rates reported in developing countries in southeast Asia [10].

This paucity and discrepancy of published data might be due to small sample sizes in studies, possibly because brain tumors are relatively less frequent than other tumors, known to account for $1-2 \%$ of the overall cancer burden [10], to different geographic repartition between regions and countries or to differences between the studies regarding the patients, the tumor types and the methods of classification of these tumors. We can add the differences in studies quality, knowing particularly that studies led in eastern countries are not based on data from nationalbased cancer registries and therefore result in biased estimates [10]. On the other hand, research in brain tumors is

Future Medicine 


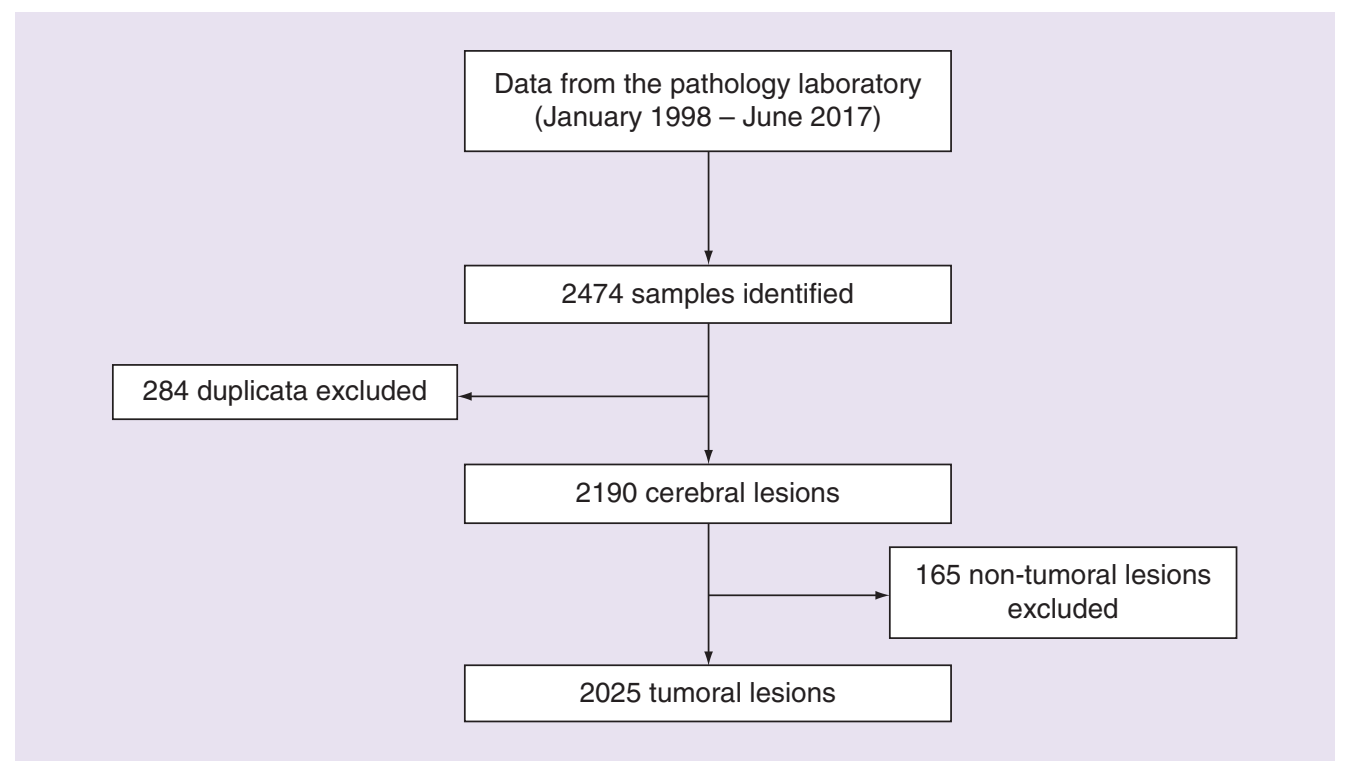

Figure 1. Collection and selection of records obtained from the pathology laboratory of Hôtel Dieu de France University hospital of Beirut from January 1998 to June 2017.

expanding worldwide after developing technologies in brain imaging, oncogenetics and the molecular biology of brain tumors [9].

In Lebanon, there are no established data concerning the different epidemiological, clinical or histological characteristics of brain tumors. The latest epidemiological data obtained in Lebanon about CNS tumors dates back only to 2015, available in the National Cancer Registry (NCR) of the Lebanese Ministry of Public Health (Beirut, Lebanon). According to the NCR, CNS tumors represent 2.19\% of all tumors, they are more common among men than women with a sex ratio M:F of 1.5 and their prevalence depends on age, with a peak between 50 and 54 years old [11].

The main purpose of this study is therefore the determination of the histological and epidemiological characteristics of brain tumors in the Lebanese population. This study would be then useful for the development of further trials in this under-explored area in Lebanon and for the improvement of health resources.

\section{Materials \& methods}

We conducted a retrospective study to evaluate the characteristics of CNS tumors. The data were collected from the records of the pathology laboratory of Hôtel Dieu de France, Saint Joseph University Hospital of Beirut (Lebanon) over a period 20 years, between January 1998 and June 2017.

The population studied was the patients of Hôtel Dieu de France hospital and the inclusion criterion was any cerebral tumor diagnosed during the specified period. The exclusion criterion was the nontumoral lesions of the brain. The variables evaluated were the sample type, the histological types and subtypes, the epidemiologic characteristics of the tumors and their clinical presentation. SPSS 24.0 was used to perform the statistical analysis.

The 2474 samples of CNS lesions were obtained from the data collected from the pathology laboratory. Duplicated data were removed, ending up with 2190 patients diagnosed over 20 years. From these patients, 165 patients were diagnosed of nontumoral lesions and were, therefore, excluded. As a result, 2025 patients with tumoral lesions were retained in the study (Figure 1).

\section{Results}

\section{Sample types}

The most common diagnostic tool was surgical resection, with a frequency of $65 \%$ (1316) of the cases. $25.4 \%$ (514) of the samples were obtained by biopsy (stereotactic biopsies, frozen section analysis or neuroendoscopy) and $3.7 \%$ (75) of them were obtained by the cerebrospinal fluid analysis. The diagnostic tool was undetermined in $5.9 \%(119)$ of the cases. 


\section{Distribution by age $\&$ sex}

The median age of diagnosis of all brain tumors was 50 years, with ages ranging from birth to 89 years old. Those diagnosed with primary CNS tumors had a median age of 49 years. The metastases diversely occurred in older patients, with a median age of 59 years. The median age of glial and nonglial tumors was 49 and 50 years, respectively.

The distribution of the tumors differed also by sex. The cerebral tumors are more common in men than women; $52.2 \%$ (1057) of the patients were men, whereas women accounted for $47.8 \%$ (968) of the patients, with a sex ratio $\mathrm{M}: \mathrm{F}$ of 1.1. The secondary tumors were more likely to occur in men corresponding to a sex ratio M:F of 1.2; however, the primary tumors occurred equally in the two sexes, corresponding therefore to a sex ratio M:F of 1.0. By histology, men had a greater rate of glial tumors (sex ratio M:F 1.5) and women, in contrast, had a greater rate of nonglial tumors (sex ratio M:F 0.6).

\section{Distribution by site}

Overall, the most common site of brain tumors was the cerebral cortex, accounting for 42.3\% (856) of all tumors with a predominance in the frontal lobe which accounted for $17.4 \%$ (352) of all tumors. The cortex was followed by the cerebellum which represented $13.6 \%$ (257) of all tumors. The meninges accounted for only $5.8 \%$ (117) of all tumors.

\section{Distribution by origin}

The rate of primary intracranial tumors registered in the pathology laboratory exceeded the rate of metastatic brain tumors approximately by a factor of 10 , with $90.7 \%$ (1837) of the tumors being primary brain tumors versus $9.3 \%$ (188) of the tumors being secondary.

\section{Distribution by histological types}

165 patients had nontumoral or pseudo-tumoral lesions (reactional tissue such as gliosis or spongiosis, cysts, vascular lesions, such as, angiomas or cavernomas, or arteriovenous malformations, inflammatory lesions and infectious lesions, such as abscesses or hydatid cysts) representing $7.5 \%$ of all lesions, and were excluded from the study. 2025 patients were found to have tumoral cerebral lesions, representing $92.5 \%$ of the records. For the 2025 patients retained, the overall rates by histology grouping are represented in Figure 2. The glial tumors accounted for the majority of the tumors with a rate of $46.2 \%$ (848) of all tumors, followed by the nonglial tumors representing $42.5 \%$ (781) of the tumors. Regarding their behavior, among the primary tumors that have definite pathologic diagnosis, $66 \%$ of the tumors were benign and $34 \%$ were malignant.

\section{Distribution by age groups \& histology}

Age group 0-14 years

9.7\% (197) of the tumoral lesions were present in this age group, with a majority of 191 lesions being primary corresponding to $97 \%$ of the cases. Among them, $56 \%$ (79) were benign and $44 \%$ (62) were malignant. Glial tumors were more frequent, accounting for $50.5 \%$ (93) of the tumors, with a predominance of astrocytic tumors accounting for $32.6 \%$ (60) with a predominance of pilocytic astrocytoma accounting for $22.8 \%$ (42) and glioblastoma accounting for $4.3 \%$ (8) of the tumors. The nonglial tumors represented approximately $39 \%$ (72) of the tumors, with a prevalence of embryonal tumors and tumors of the sellar region accounting approximately for $24 \%$ (44) and $8.2 \%$ (15) of the tumors, respectively. The embryonal tumors were mostly medulloblastoma, with a frequency of $15.8 \%(29)$, and the tumors of the sellar region were all craniopharyngioma.

Age group 15-39 years

This age group represented around 22\% (444) of the tumors. Among the well-defined tumors, $77.7 \%$ (251) and $22.3 \%$ (72) were benign and malignant, respectively. Only $3 \%(14)$ of the tumors were secondary. $48 \%$ (199) of the primary tumors were glial and 39\% (165) were nonglial. Within the glial histologic group, astrocytic and oligodendroglial tumors were equally present, each with a prevalence of $16.7 \%$ (69) of the cases. Each of the pilocytic astrocytoma and glioblastoma represented 5\% (20) among the astrocytic tumors. Oligodendroglial tumors were mostly oligodendroglioma, accounting for $15.2 \%$ (63) of the tumors. Whereas, the meningeal tumors, tumors of the cranial and paraspinal nerves and embryonal tumors were the most frequent subtypes of glial tumors with a prevalence of $14.3 \%$ (59), $10.9 \%$ (45) and $8.5 \%$ (35) of the cases, respectively. Meningioma represented 


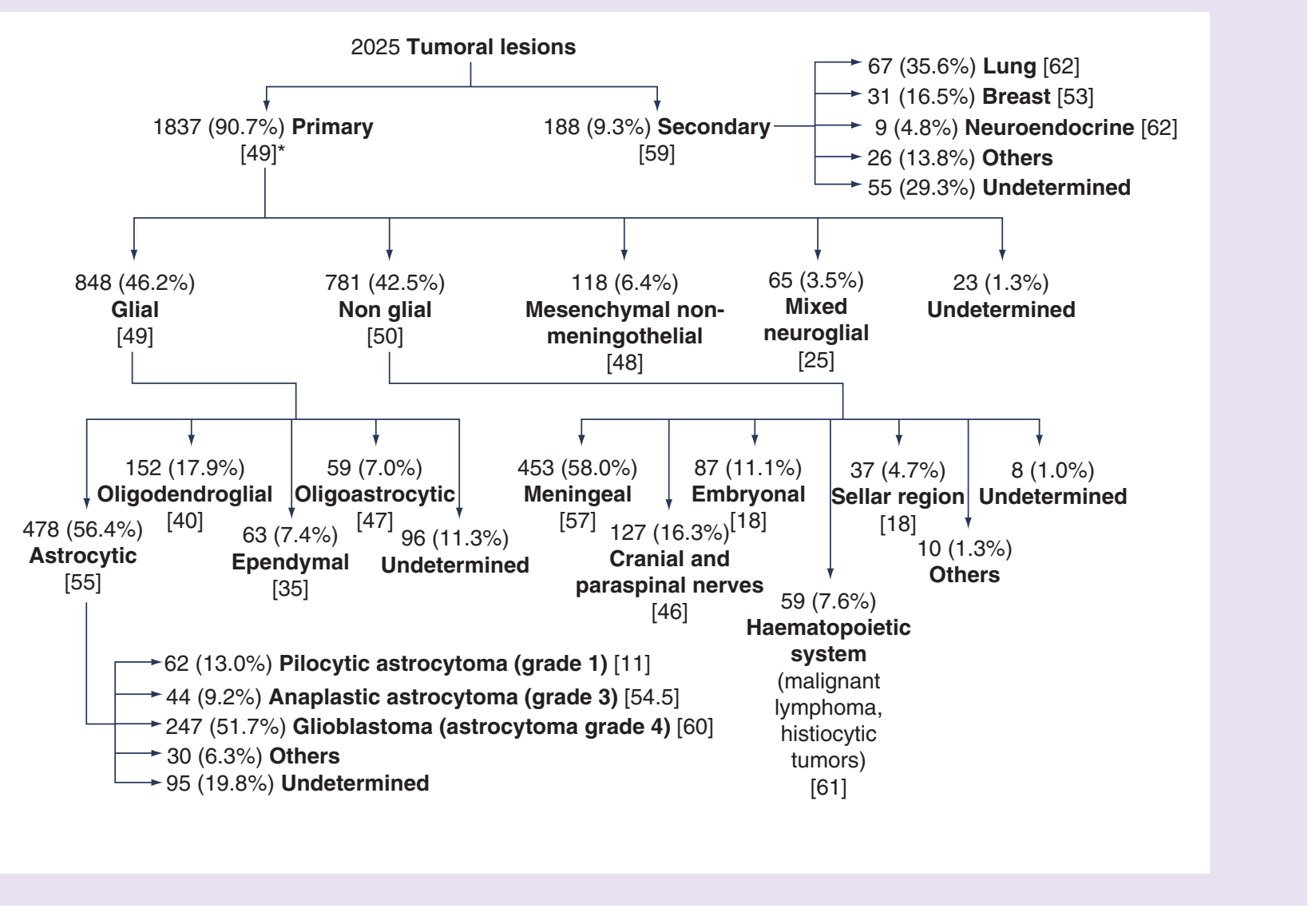

Figure 2. Distribution of histologic subtypes of all brain tumoral lesions collected from the pathology laboratory of Hôtel Dieu de France University hospital of Beirut from January 1998 to June 2017.

*Median age is represented in years between square brackets.

the majority of meningeal tumors, accounting for $11.4 \%$ (47) of the tumors with the greater prevalence for the meningothelial meningioma representing 5\% (21) of the cases. The major histologic subtype of tumors of the cranial and paraspinal nerves was schwannoma with a frequency of $10.4 \%$ (43) and medulloblastoma was the major subtype of embryonal tumors with a frequency of $7 \%$ (29) of the tumors.

Age group 40 years \& older

The great majority of tumors - 68\% (1384) of the cases - were found in this age group, with the majority being benign tumors representing 64\% (605) of the tumors whereas 36\% (341) were malignant. Glial and nonglial tumors were almost equally frequent with a prevalence of $42.7 \%(552)$ and $42 \%$ (541), respectively. However, in this age group, the prevalence of secondary brain tumors was $12 \%$ (168).

Astrocytic tumors were the most frequent of the glial tumors with a frequency of $27 \%$ (349) and a predominance of glioblastoma representing $17.3 \%$ (223) of the tumors. In the same manner, meningeal tumors were the most frequent among glial tumors, accounting for $30.3 \%$ (392) of the cases with a predominance of meningioma representing $29.4 \%$ (380) of the tumors and with the meningothelial being the most frequently reported subtype representing $11 \%$ (142) of the cases.

Evolution through years

The distribution of cases collected through the duration of 20 years is represented in Table 1. 
Table 1. Distribution of brain and CNS tumors collected in the pathology laboratory of Hôtel Dieu University hospital of Beirut through two decades.

\begin{tabular}{|c|c|c|c|c|}
\hline Year-interval & 1999-2003 & 2004-2008 & 2009-2013 & 2014-2017 \\
\hline Total & 202 & 648 & 709 & 466 \\
\hline Primary & $184(90 \%)$ & $588(90.7 \%)$ & $643(90 \%)$ & $422(90 \%)$ \\
\hline Secondary & $13(6.4 \%)$ & $54(8 \%)$ & $70(9.8 \%)$ & $51(10 \%)$ \\
\hline Glial & $127(63 \%)$ & $254(39 \%)$ & $280(39.5 \%)$ & $187(40 \%)$ \\
\hline Astrocytic & $72(36 \%)$ & $115(18 \%)$ & $172(24 \%)$ & $119(25.5 \%)$ \\
\hline Oligodendroglial & $23(11.4 \%)$ & $50(7.7 \%)$ & $53(7.5 \%)$ & $26(5.6 \%)$ \\
\hline Ependymal & $11(5.4 \%)$ & $21(3.2 \%)$ & $12(1.7 \%)$ & $18(4 \%)$ \\
\hline Oligoastrocytic & $9(4.4 \%)$ & $22(3.4 \%)$ & $23(3.2 \%)$ & $5(1 \%)$ \\
\hline Nonglial & $32(15.3 \%)$ & $269(41.5 \%)$ & $285(40.2 \%)$ & $195(42 \%)$ \\
\hline Meningeal & $8(4 \%)$ & $166(25.6 \%)$ & $163(23 \%)$ & $116(25 \%)$ \\
\hline Cranial and paraspinal nerves & 0 & $36(5.5 \%)$ & $52(7.3 \%)$ & $39(8.3 \%)$ \\
\hline Embryonal & $16(7.4 \%)$ & $31(4.6 \%)$ & $28(4 \%)$ & $12(2.5 \%)$ \\
\hline $\begin{array}{l}\text { Tumors of the hematopoietic } \\
\text { system }\end{array}$ & $7(3.4 \%)$ & $14(2 \%)$ & $19(2.6 \%)$ & $19(4 \%)$ \\
\hline Tumors of the sellar region & $2(1 \%)$ & $18(2.7 \%)$ & $11(1.5 \%)$ & $6(1.3 \%)$ \\
\hline
\end{tabular}

Table 2. Distribution of primary glial brain and CNS tumors diagnosed at Hôtel Dieu de France University hospital of Beirut from 1998 to June 2017 by histology, age and sex.

\begin{tabular}{|c|c|c|c|c|c|c|}
\hline \multirow[t]{2}{*}{ Primary glial tumors } & \multirow[t]{2}{*}{ Mean age (years) } & \multicolumn{2}{|c|}{$\operatorname{Sex}(\%)$} & \multicolumn{2}{|c|}{$\operatorname{Sex}(n)$} & \multirow[t]{2}{*}{ Total } \\
\hline & & Males & Females & Males & Females & \\
\hline Astrocytic tumors & 49 & 59.1 & 40.9 & 196 & 282 & 478 \\
\hline $\begin{array}{l}\text { Oligodendroglial } \\
\text { tumors }\end{array}$ & 41 & 55.7 & 44.3 & 85 & 67 & 152 \\
\hline Ependymal tumors & 33 & 63.9 & 36.1 & 40 & 23 & 63 \\
\hline $\begin{array}{l}\text { Oligoastrocytic } \\
\text { tumors }\end{array}$ & 46 & 69.5 & 30.5 & 41 & 17 & 59 \\
\hline Overall glial tumors & 46 & 60.5 & 39.5 & 513 & 335 & 848 \\
\hline
\end{tabular}

\section{Distribution of glial CNS tumors \\ By histology}

Among primary glial brain tumors, the astrocytic tumors were the most frequent histological subtype, accounting for $56.4 \%$ (478) of glial tumors, followed by oligodendrogial tumors, accounting for $17.9 \%$ (152) of glial tumors.

In the histological subtype of astrocytic tumors, the malignant tumors were the most common tumors. The grade IV astrocytoma (the glioblastoma), represented more than the half of the cases with a rate of 51.7\% (247) of the astrocytic tumors, as seen in Figure 2, followed by the pylocytic astrocytoma and the anaplastic astrocytoma accounting for 13\% (62) and 9.2\% (44) of the astrocytic tumors, respectively. Therefore, the glioblastoma accounted for $13.4 \%$ of all primary tumors.

The rates of the other different histological subtypes of glial tumors are represented in Figure 2.

By age

The overall mean and median ages of diagnosis of primary glial brain tumors were 46 and 49 years, respectively. The mean age for each histological subtype is shown in Table 2.

All the glial tumors occurred more predominantly in men than in women with an overall prevalence of $60.5 \%$ (513) of glial tumors reported in men. The prevalence rates according to sex for the histological subtypes of glial tumors are shown in Table 2. 
Table 3. Distribution of primary nonglial brain and CNS tumors diagnosed at Hôtel Dieu de France University hospital of Beirut from January 1998 to June 2017 by type and histological subtype.

\begin{tabular}{|c|c|c|c|c|}
\hline Tumor cell of origin & Frequency, \% (n) & Histological subtype & Tumors within cell of origin $\%$ & All nonglial tumors $\%$ \\
\hline \multirow[t]{3}{*}{ Meningeal tumors } & $58(453)$ & Meningioma & 94.7 & 54.5 \\
\hline & & Hemangioblastoma & 3.5 & 2 \\
\hline & & Others & 1.8 & \\
\hline \multirow{3}{*}{$\begin{array}{l}\text { Cranial nerves \& paraspinal } \\
\text { tumors }\end{array}$} & $16.3(127)$ & Schwannoma & 96 & 15.5 \\
\hline & & Neurofibroma & 0.8 & 0.1 \\
\hline & & Others & 3.2 & \\
\hline \multirow[t]{6}{*}{ Embryonal tumors } & $11.1(87)$ & Medulloblastoma & 74.7 & 8.3 \\
\hline & & PNETS & 11.5 & 1.3 \\
\hline & & $\begin{array}{l}\text { Atypical teratoid/rhabdoid } \\
\text { tumors }\end{array}$ & 5.7 & 0.64 \\
\hline & & Ependymoblastoma & 3.4 & 0.4 \\
\hline & & Medulloepithelioma & 1.1 & 1.3 \\
\hline & & Others & 3.6 & \\
\hline \multirow{2}{*}{$\begin{array}{l}\text { Tumors of the hematopoietic } \\
\text { system }\end{array}$} & $7.6(59)$ & Malignant lymphoma & 95 & 7.2 \\
\hline & & Histiocytic tumors & 5 & 0.5 \\
\hline \multirow[t]{2}{*}{ Tumor of the sellar region } & $4.7(37)$ & Craniopharyngioma & 97.3 & 4.6 \\
\hline & & Others & 2.7 & \\
\hline Undetermined & $2.3(8)$ & & & \\
\hline
\end{tabular}

By site

The frontal and temporal lobes were the preferential sites for the primary glial tumors representing the site of $39 \%$ (330) of the reported tumors. This predilection for the frontal and temporal lobes was seen also in all the histological subtypes of glial tumors except for the ependymal tumors which had their preferential site in the spinal cord accounting for $41 \%$ (347) of the tumors.

\section{Distribution of nonglial CNS tumors By cell of origin}

Meningeal tumors are the most frequently reported nonglial brain tumors, accounting for 58\% (453) of these tumors (Table 3).

\section{By histology}

Among the meningeal tumors, the meningioma was the most common histological subtype, representing $94 \%$ (426) of the tumors. Therefore, the meningioma is qualified as the most frequent histological type of nonglial tumors, accounting for $54.5 \%$ of all the nonglial tumors. In parallel, the meningioma accounts for $23 \%$ of all primary tumors.

The meningothelial was the most frequent subtype of meningioma, accounting for 39\% (166) of meningiomas. The distribution of meningiomas' histological subtypes is represented in Figure 3A.

The other histological subtypes distribution of nonglial tumors by site is detailed in Table 3 .

By sex

The nonglial tumors were more common in women with a sex ratio M:F of all nonglial primary CNS tumors of 0.6. This prevalence in women was found in all meningiomas' subtypes, except for the atypical meningioma which was more frequently reported in men than women (Figure 3B).

By age

The mean and median ages of nonglial tumors were 47 and 50 years, respectively. The age of all primary nonglial CNS tumors was between 40 and 50 years old; the mean age of meningioma, schwannoma and malignant lymphoma 
(A)

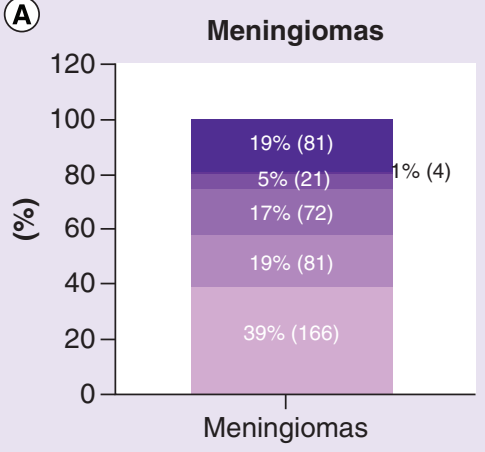

- Others

- Microcystic [60 $]^{*}$

- Atypical [59]

- Fibroblastic [56]

- Transitional $[57.5]^{*}$

- Meningothelial $[55]^{*}$
(B)

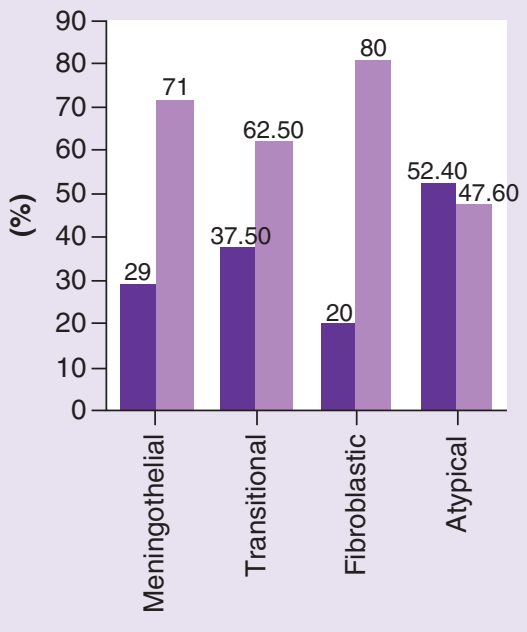

- Men - Women

Figure 3. Distribution by (A) histological subtypes and (B) sex of meningiomas diagnosed at Hôtel Dieu de France University hospital of Beirut from January 1998 to June 2017. *Median age is represented in years between square brackets.

was 56, 45.2 and 57.4 years, respectively. However, the medulloblastoma and the craniopharyngioma occurred at a younger age, with a mean age of diagnosis around 20 years old.

\section{Distribution of metastatic brain lesions \\ By origin}

Lung cancer was the most common cancer reported to have metastases to the brain, accounting for $35.6 \%$ (67) of all the secondary lesions. Breast cancer was the second most common cancer reported for 16.5\% (31) of the metastases. Neuroendocrine tumors were responsible for $4.8 \%$ (nine) of brain metastases. The origin was undetermined in $29 \%$ (55) of the cases, based on the interpretation of the tissue sample by the pathologist who qualified the corresponding tissues as 'undetermined' according to anatomopathological features.

By site

The most frequent site of brain metastases was the cortex accounting for approximately $50 \%$ (94) of the localizations, with the frontal lobe representing $23.4 \%$ (44) of the tumors site and being therefore the most common site of intracranial metastases. The cortex is followed by the cerebellum accounting for $18.6 \%$ (35) of the tumors.

By age \& sex

The mean age of brain metastases was 58 years and brain metastases were more frequently reported in men than women; $53.3 \%$ (101) of the metastases occurred in men while $44.7 \%$ (87) occurred in women.

\section{Revealing symptoms}

The most frequent clinical symptom of brain tumors was epilepsy, occurring in $22.5 \%$ (455) of the cases, followed by headache, representing 20.6\% (417) of the tumors, and motor deficit, representing 19.9\% (403). Other reported revealing clinical symptoms include dizziness, aphasia or dysarthria, and visual problems occurring in $12 \%$ (243), $9.2 \%$ (186) and $7.7 \%$ (156) of the cases respectively. To note that the data were only collected from the clinical information written by the surgeon on the pathology form sent with the samples to the pathology laboratory. 


\section{Discussion}

According to the Lebanese NCR-2015, the brain and CNS tumors account for $2.19 \%$ of all cancers reported, with age-standardized incidence rates of brain and CNS tumors of 6.9 per 100,000 population in men and 4.4 per 100,000 population in women, but no other information concerning other epidemiologic characteristics or detailing histologic subtypes. The NCR registered 285 incident cases in both genders during the same year; the incidence regardless of gender can therefore be estimated at 4.36 per 100,000 population [11]. This prevalence is met in other studies, especially in the CBTRUS (Central Brain Tumor Registry of the United States) report where the prevalence of brain and CNS tumors is $2 \%$ of all cancers [10,12].

In comparison with the American population where one of the biggest statistical studies is held by the CBTRUS and includes more than 390,000 patients, our population is characterized by an overall median age of all primary brain tumors younger than the studied American population by 10 years [1]. Similar to what is found in the literature, the prevalence of brain tumors increases in older age [1,13], especially in the adult age group of 40 years and older. Specifically, some histologic subtypes mentioned in Figure 2 have an approximately 10 -year earlier median age at diagnosis compared with the Western literature, such as ependymal tumors, meningeal tumors, cranial and paraspinal nerves tumors and sellar region tumors. Importantly, our population included patients who consulted the Hôtel Dieu de France hospital, one of the biggest tertiary centers in Beirut. Therefore, many patients would have been referred to the hospital from different Lebanese regions and would expectedly be younger than the patients who would be treated from their diagnosis to their remission or death in the hospitals available in their regions, especially the elder patients whose prognosis is poor. This fact could explain the difference in age found in our study compared with the literature.

It has been shown that cerebral tumors are more common among women $[1,8]$. However, in our population, another different characteristic is that the brain tumors occur more frequently in men than women, with a sex ratio M:F of 1.1. Particularly, the M:F ratio for primary brain tumors in our population is 1.0 versus a M:F ratio of 0.7 for primary brain tumors in the American population according to the CBTRUS report [1]. This higher prevalence in women in the American population is attributable to the higher frequency of meningiomas shown in the CBTRUS report, while the meningioma in our population is less frequent. The higher prevalence among men in our study is true except for the category of nonglial brain tumors where the sex ratio M:F was 0.6. This exception may be attributable to the known predominance of the meningioma among women $[1,12,14]$ that is also met in our population. Although we found this difference, we can attribute it to the population selected in our study. As we mentioned before, many of the patients included in these results would be referred from other hospitals in other regions of Lebanon. On the other hand, it is known by neurosurgeons that the meningioma is relatively an easy tumor to treat because of its benign nature and its localization that is, usually, easily reachable by surgery. Given this, a good number of meningiomas could be treated and resected in other Lebanese regions without their transfer to our hospital, leading to this lower prevalence of meningiomas in our population.

By behavior, primary tumors in our population have the same pattern of prevalence as the prevalence found in the CBTRUS report: benign and malignant tumors in our study accounted for $66 \%$ and $34 \%$, respectively, compared with 68.5 and $31.5 \%$, respectively, in the CBTRUS report. Although benign tumors were predominant in all age groups, the greatest frequency of malignant tumors is observed in the age group $0-14$ years. This highest frequency is also observed in the CBTRUS report, in which it also surpasses the frequency of benign tumors [1].

By age groups, especially for the age group 0-14 years, our population presents a higher prevalence of brain and CNS tumors given that $9.7 \%$ of the tumors were present in this age group in our study versus $5 \%$ in the same age group in the American population studied by the CBTRUS team. Besides, the prevalence of glial tumors of $50.5 \%$ of the tumors meets the prevalence of this histologic type in the CBTRUS report: $52.8 \%$ [1]. Other comparisons include a slightly higher prevalence in our study of pilocytic astrocytoma, glioblastoma, embryonal tumors and craniopahryngioma accounting respectively for $22.8,4.3,24$ and $8.2 \%$ of the cases against $18.2,2.8,13.5$ and $3.9 \%$ in the CBTRUS report. The higher prevalence of medulloblastoma among embryonal tumors is also met with a frequency of $66 \%$ of embryonal tumors in our study and $63 \%$ in the CBTRUS report [1]. According to the other age groups, the greatest prevalence of the tumors of the pituitary region in young adults [1] is not met in our study, giving its place for glial tumors. However, as mentioned in the literature [1,7,14], the meningioma is the major histologic subtype of tumors found in older patients.

By site, the most frequent reported anatomical site of brain tumors in our study was the cortex with a predominance in the frontal lobe accounting for $42.3 \%$ and $17.4 \%$ respectively. In contrast, according to the CBTRUS 
report, the meninges were the most common site of brain and CNS tumors representing $36.8 \%$ of the cases [1], though in our study the meninges represent only $5.8 \%$ of all the cases. In fact, this percentage could not be completely reliable because the data concerning the site of the tumors was obtained mainly in reference to the clinical information written by the surgeons on the pathology form accompanying the samples sent to the pathology laboratory where the clinical information is usually not highly accurate, especially in determining the site of the sample. For example, a meningeal tumor in the frontal area may be written by the surgeon as a frontal tumor. Therefore, some meningeal tumors may be lost or attributed to other localizations, leading to this underestimation of the prevalence of brain tumors in the meninges.

Among primary brain tumors, our population represented a higher prevalence of glial tumors (46.2\%), whereas, according to the CBTRUS report, the glial tumors account for only $26 \%$ of all primary brain tumors [1]. On the other hand, similarly to the results in the CBTRUS report where the glioblastoma accounted for the majority of primitive gliomas (56.6\%) [1], the glioblastoma is the most frequent histological subtype in primary glial tumors reported in our study accounting for $51.7 \%$ of astrocytic primary tumors and for $29.1 \%$ of all primary glial tumors. Similarly, the meningioma occupies an important place among nonglial tumors in being the most common histological type among nonglial tumors $(54.5 \%)$ and in meningeal tumors $(94.7 \%)$ and therefore it is the most reported histological subtype accounting for $23 \%$ of all primary tumors as in the CBTRUS report where the meningioma accounted for $37.1 \%$ of all tumors [1] but with a lower percentage, as explained above.

Other similarities include the higher prevalence of glial tumors within the cortex [1] and in men [1,9]. Although, according to the meta-analysis held by de Robles et al., the difference by sex for histological types of brain tumors has been shown to be statistically nonsignificant on the overall estimate of brain tumors, except for the pooled incidence rate of meningioma that was borderline significant with an estimate for women higher than that for men (4.21 per 100,000 person-years for women versus 2.33 per 100,000 person-years for men with a $p=0.05$ ) [8].

Regarding metastases, they constitute one of the most important neurological mishaps of cancers and their incidence is increasing [15]. In fact, the incidence of brain tumors and brain metastases has been reported to be increasing lately due to better diagnostic abilities including the advanced technologies in brain imaging (such as CT-Scan and MRI), the increasing number of specialists and neurologists, better access to medical care, the newly available therapies and so on $[8,9,15,16$. All these factors allow for prolonged life expectancy and further diagnosis of primary and secondary brain tumors.

Therefore, brain metastases are ten-times more frequent than primary brain tumors and can account for more than $50 \%$ of intracranial tumors $[15,17-19]$. However, in our study the rate of primary brain tumors exceeded by ten-times the rate of metastatic lesions. This result does not contrast with the known predominance of metastases over primary tumors, but the data in our study do not include other than the pathological diagnosis of brain tumors, therefore the radiological and the clinical diagnoses which contribute by themselves to a further diagnosis of brain metastases are not reported.

The prevalence of brain metastases increases with age, corresponding to their occurrence in the late course of the primary disease. As published in previous studies [15], the most common site of brain metastases in our study was the cerebral cortex. Although any cancer can disseminate to the brain [15], the most common primary sites for brain metastases were lung and breast cancer [15,20-31]. By sex, our results met those found in previous studies stating that brain metastases are more frequent among men than women. This difference is explained by the prevalence of lung cancer in men reported in the literature [17] and valid in the Lebanese population, where $67 \%$ of lung tumors occur in men according to the Lebanese NCR-2015 [11].

Regarding the evolution of the collected cases through the duration of two decades of our study, it is remarkable that a higher number of cases is collected starting the year 2004 and maintained for the next 10 years, especially among the nonglial histologic type and particularly meningeal tumors and cranial and paraspinal nerves tumors, and the secondary lesions - despite being a modest elevation in the latter cases. This variation could be due to the progression in clinical practice and advanced diagnostic facilities, as discussed above, and the recruitment of more neurologists and neurosurgeons in our institution. However, the decrease of total number of cases in the last interval could be attributable to an unequal duration of this interval compared with the previous ones. However, the proportions of histologic subtypes and the slightly greater number of cases of secondary lesions were still maintained.

With respect to revealing symptoms, the most encountered symptoms in our study, i.e. epilepsy, headache and motor deficit, join the symptoms presented in the literature [16,32-44]. The most reported known symptoms of brain tumors are headache and seizures [45]. Headache is a common symptom of brain tumors that has been shown to 
be present in almost half of the cases and are the presenting symptom in approximately $25 \%$ of the patients [37,38]. In parallel, headache in our study was the revealing symptom in $20.6 \%$ of the cases. Only $22.5 \%$ of our cases had seizures as the revealing symptoms, whereas the literature report a greater frequency. A previous retrospective study showed that seizures were the presenting symptom in approximately $40 \%$ of primary brain tumors and $20 \%$ of brain metastases [46] and in another prospective registry study, they were the presenting symptom in approximately $70 \%$ of primary brain tumors [47].

The first limitation of our study is the quality of patients included who would be, in great probability, difficult cases concerning young patients or patients encountering difficulties in the mean of treatment. This type of patient would be a justification to refer them to tertiary center such as Hôtel Dieu de France Hospital of Beirut. The second limitation is the missing data in some fields, leading to a lack of some features from the statistical counts, especially that the data does not include the patients whose brain tumors, primary or particularly secondary, are diagnosed by imaging without biopsy. This weak point would be referred to a nonstandardized method of registering the brain tumors when diagnosed by oncologists or neurosurgeons or pathologists or radiologists.

\section{Conclusion \& future perspective}

This study is the first of its kind in our country, determining the epidemiological characteristics of brain tumors in the Lebanese population. Despite meeting many epidemiological aspects known in the literature, a younger age of onset, a lower prevalence of meningiomas and a higher prevalence of gliomas were noticed in the Lebanese population. This study is essential in order to build more trials and develop further research in this insufficiently explored field in Lebanon. Using our data, we would be able to evaluate the prognosis, treatment and survival characteristics in each subgroup of tumors types and compare the histologic diagnosis of oligodendroglioma to molecular diagnosis by using codeletion 1p19q and other molecular biology tests (such as IDH1, IDH2).

\section{Summary points}

- In Lebanon, there are no established data concerning the different epidemiological, clinical or histological characteristics of brain tumors.

- We conducted a retrospective study to evaluate the characteristics of CNS tumors for the patients found in the records of the pathology laboratory of Hôtel Dieu de France, Saint Joseph University hospital of Beirut over 20 years, between January 1998 and June 2017, including a total of 2025 cases.

- The median age of diagnosis of all tumors was 50 years. Tumors were more frequently reported in men, representing $52.2 \%$ of the tumors.

- The most frequent localization reported was the cortex, accounting for $42.3 \%$ of the cases.

- The majority of the tumors were glial tumors, accounting for $46.2 \%$ of the cases whereas nonglial tumors represented $42.5 \%$ of them.

- Among glial tumors, astrocytic tumors were the most frequent subtype, presenting $56.4 \%$ of the cases with predominance of the glioblastoma accounting for $51.7 \%$ of astrocytic tumors. The median age of diagnosis was 49 years. The sex ratio M:F was at 1.5. The most common localization was the frontal and temporal lobes in $39 \%$ of the cases.

- Among nonglial tumors, meningeal tumors presented $58 \%$ of the cases, with predominance of meningioma accounting for $94 \%$ of the tumors. The median age of diagnosis was 50 years. The sex ratio M:F was at 0.6.

- In the metastatic lesions, lung cancer was the first primary accounting for $35.6 \%$ of the cases, followed by the breast in $16.5 \%$ of the cases. The metastases were found predominantly in the cortex. The mean of age of diagnosis of secondary tumors was 58 years. Metastases were more frequent in women than men with a prevalence of $53.3 \%$.

- The revealing symptoms of CNS tumors were primarily epilepsy $(22.5 \%)$, headache $(20.6 \%)$ and motor deficit $(19.9 \%)$.

- This is the first epidemiologic study concerning brain and CNS tumors in Lebanon, which is essential to build more trials in this field.

\section{Acknowledgments}

This study is one of the research activities of the department of Hematology-Oncology in collaboration with the department of Pathology of Hôtel Dieu de France, Saint Joseph University Hospital in Beirut. The authors wish to acknowledge all the members of these two departments for their contribution to the success of this study. 
Financial \& competing interests disclosure

The authors have no relevant affiliations or financial involvement with any organization or entity with a financial interest in or financial conflict with the subject matter or materials discussed in the manuscript. This includes employment, consultancies, honoraria, stock ownership or options, expert testimony, grants or patents received or pending, or royalties.

No writing assistance was utilized in the production of this manuscript.

\section{Open access}

This work is licensed under the Attribution-NonCommercial-NoDerivatives 4.0 Unported License. To view a copy of this license, visit http://creativecommons.org/licenses/by-nc-nd/4.0/

\section{References}

Papers of special note have been highlighted as: $\bullet$ of interest; $\bullet \bullet$ of considerable interest

1. Ostrom QT, Gittleman H, Truitt G, Boscia A, Kruchko C, Barnholtz-Sloan JS. CBTRUS statistical report: primary brain and other central nervous system tumors diagnosed in the United States in 2011-2015. Neuro Oncol. 20(Suppl. 4), iv1-iv86 (2018).

-. This is one of the biggest studies concerning the epidemiologic characteristics of brain and CNS tumors held in the USA that could be found in the literature.

2. Louis DN, Ohgaki H, Wiestler OD et al. The 2007 WHO classification of tumours of the central nervous system. Acta Neuropathol. 114(2), 97-109 (2007).

3. Louis DN, Perry A, Reifenberger G et al. The 2016 World Health Organization classification of tumors of the central nervous system: a summary. Acta Neuropathol. (Berl). 131(6), 803-820 (2016).

4. Jacques G, Cormac O. Central nervous system tumors. Handb. Clin. Neurol. 112, 931-958 (2013).

5. Lacy J, Saadati H, Yu JB. Complications of brain tumors and their treatment. Hematol. Oncol. Clin. North Am. 26(4), 779-796 (2012).

6. Street W. Cancer Facts \& Figures 2018. 76 (1930). www.cancer.org/research/cancer-facts-statistics/all-cancer-facts-figures/ cancer-facts-figures-2018.html

7. McNeill KA. Epidemiology of brain tumors. Neurol. Clin. 34(4), 981-998 (2016).

8. de Robles P, Fiest KM, Frolkis AD et al. The worldwide incidence and prevalence of primary brain tumors: a systematic review and meta-analysis. Neuro Oncol. 17(6), 776-783 (2014).

- This article is one of the rare studies elucidating the worldwide incidence of primary brain tumors, which is rarely studied.

9. Bondy ML, Scheurer ME, Malmer B et al. Brain tumor epidemiology: consensus from the Brain Tumor Epidemiology Consortium. Cancer 113(S7), 1953-1968 (2008).

10. Gupta T, Achari R, Chatterjee A et al. comparison of epidemiology and outcomes in neuro-oncology between the East and the West: challenges and opportunities. Clin. Oncol. 31(8), 539-548 (2019).

11. Lebanese Ministry of Public Health (MOPH). National Cancer Registry 2015 (2015). www.moph.gov.lb/en/Pages/8/19526/ national-cancer-registry

-. This site is the only epidemiologic source for brain and CNS tumors available in Lebanon.

12. Ostrom QT, Gittleman H, Liao P et al. CBTRUS statistical report: primary brain and central nervous system tumors diagnosed in the United States in 2007-2011. Neuro Oncol. 16(Suppl. 4), iv1-iv63 (2014).

13. Nayak L, Iwamoto FM. Primary brain tumors in the elderly. Curr. Neurol. Neurosci. Rep. 10(4), 252-258 (2010).

14. Dolecek TA, Dressler EVM, Thakkar JP, Liu M, Al-Qaisi A, Villano JL. Epidemiology of meningiomas post-Public Law 107-206: the benign brain tumor cancer registries amendment act. Cancer 121(14), 2400-2410 (2015).

15. Nayak L, Lee EQ, Wen PY. Epidemiology of brain metastases. Curr. Oncol. Rep. 14(1), 48-54 (2012).

-• Shows the frequency of brain metastases and their most common primary tumors.

16. Butowski NA. Epidemiology and diagnosis of brain tumors. Continuum (Minneap. Minn.) 21(2 Neuro-oncology), 301-313 (2015).

-. Gives information on some epidemiologic characteristics of primary and secondary brain and CNS tumors including symptoms features.

17. Walker AE, Robins M, Weinfeld FD. Epidemiology of brain tumors: the national survey of intracranial neoplasms. Neurology 35(2), 219-226 (1985).

18. Cage TA, Mueller S, Haas-Kogan D, Gupta N. High-grade gliomas in children. Neurosurg. Clin. N. Am. 23(3), 515-523 (2012).

19. Percy AK, Elveback LR, Okazaki H, Kurland LT. Neoplasms of the central nervous system. Epidemiologic considerations. Neurology 22(1), 40-48 (1972).

20. Diaz ME, Debowski M, Hukins C, Fielding D, Fong KM, Bettington CS. Non-small cell lung cancer brain metastasis screening in the era of positron emission tomography-CT staging: current practice and outcomes. J. Med. Imaging Radiat. Oncol. 62(3), 383-388 (2018). 
21. Zavitsanos PJ, Wazer DE, Hepel JT, Wang Y, Singh K, Leonard KL. BRCA1 mutations associated with increased risk of brain metastases in breast cancer: a 1: 2 matched-pair analysis. Am. J. Clin. Oncol. 41(12), 1252-1256 (2018).

22. Vosoughi E, Lee JM, Miller JR et al. Survival and clinical outcomes of patients with melanoma brain metastasis in the era of checkpoint inhibitors and targeted therapies. BMC Cancer 18(1), 490 (2018).

23. Tio M, Wang X, Carlino MS et al. Survival and prognostic factors for patients with melanoma brain metastases in the era of modern systemic therapy. Pigment Cell Melanoma Res. 31(4), 509-515 (2018).

24. Keogh BP, Henson JW. Clinical manifestations and diagnostic imaging of brain tumors. Hematol. Clin. 26(4), 733-755 (2012).

25. DeAngelis LM, Posner JB. Neurologic complications of cancer. Oxford University Press, NY, USA (2008).

26. Counsell CE, Collie DA, Grant R. Incidence of intracranial tumours in the Lothian region of Scotland, 1989-1990. J. Neurol. Neurosurg. Psychiatry 61(2), 143-150 (1996).

27. Fabi A, Felici A, Metro G et al. Brain metastases from solid tumors: disease outcome according to type of treatment and therapeutic resources of the treating center. J. Exp. Clin. Cancer Res. 30(1), 10 (2011).

28. Stark AM, Stöhring C, Hedderich J, Held-Feindt J, Mehdorn HM. Surgical treatment for brain metastases: prognostic factors and survival in 309 patients with regard to patient age. J. Clin. Neurosci. 18(1), 34-38 (2011).

29. Graf A-H, Buchberger W, Langmayr H, Schmid KW. Site preference of metastatic tumours of the brain. Virchows Arch. A. 412(5), 493-498 (1988).

30. Lagerwaard F, Levendag P, Nowak PC, Eijkenboom WH, Hanssens PJ, Schmitz PM. Identification of prognostic factors in patients with brain metastases: a review of 1292 patients. Int. J. Radiat. Oncol. Biol. Phys. 43(4), 795-803 (1999).

31. Nussbaum ES, Djalilian HR, Cho KH, Hall WA. Brain metastases: histology, multiplicity, surgery, and survival. Cancer 78(8), 1781-1788 (1996).

32. Kirby S, Purdy RA. Headaches and brain tumors. Neurol. Clin. 32(2), 423-432 (2014).

- Discusses the importance of headache as a main symptom related to brain tumors.

33. Schiff D, Lee EQ, Nayak L, Norden AD, Reardon DA, Wen PY. Medical management of brain tumors and the sequelae of treatment. Neuro Oncol. 17(4), 488-504 (2014).

- Discusses the importance of seizures as a main symptom related to brain tumors.

34. Posti JP, Bori M, Kauko T et al. Presenting symptoms of glioma in adults. Acta Neurol. Scand. 131(2), 88-93 (2015).

•• Exposes the most common presenting symptoms of gliomas with emphasis on seizures, cognitive disorder and headache.

35. Buckner JC, Brown PD, O’Neill BP, Meyer FB, Wetmore CJ, Uhm JH. Central nervous system tumors. Mayo Clin. Proc. 82(10), 1271-1286 (2007).

36. Chandana SR, Movva S, Arora M, Singh T. Primary brain tumors in adults. Am. Fam. Physician 77(10), 1423-1430 (2008).

37. Forsyth PA, Posner JB. Headaches in patients with brain tumors A study of 111 patients. Neurology 43(9), 1678-1678 (1993).

38. Pfund Z, Szapary L, Jaszberenyi O, Nagy F, Czopf J. Headache in intracranial tumors. Cephalalgia 19(9), 787-790 (1999).

39. Valentinis L, Tuniz F, Valent F et al. Headache attributed to intracranial tumours: a prospective cohort study. Cephalalgia 30(4), 389-398 (2009).

40. Stovner LJ, Zwart J-A, Hagen K, Terwindt GM, Pascual J. Epidemiology of headache in Europe. Eur. J. Neurol. 13(4), 333-345 (2006).

41. Schankin CJ, Ferrari U, Reinisch VM, Birnbaum T, Goldbrunner R, Straube A. Characteristics of brain tumour-associated headache. Cephalalgia 27(8), 904-911 (2007).

42. Vazquez-Barquero A, Ibanez FJ, Herrera S, Izquierdo JM, Berciano J, Pascual J. Isolated headache as the presenting clinical manifestation of intracranial tumors: a prospective study. Cephalalgia 14(4), 270-271 (1994).

43. Bromfield EB. Epilepsy in patients with brain tumors and other cancers. Rev. Neurol. Dis. 1(Suppl. 1), S27-S33 (2004).

44. vanBreemen MS, Wilms EB, Vecht CJ. Epilepsy in patients with brain tumours: epidemiology, mechanisms, and management. Lancet Neurol. 6(5), 421-430 (2007).

45. Shapiro WR. Clinical features: neurology of brain tumor and paraneoplastic disorders. In: Youmans Neurological Surgery (6th Edition). Winn HR (Ed.). Elsevier Saunders, PA, USA 1197-1205 (2011).

46. Lynam LM, Lyons MK, Drazkowski JF et al. Frequency of seizures in patients with newly diagnosed brain tumors: a retrospective review. Clin. Neurol. Neurosurg. 109(7), 634-638 (2007).

47. Michelucci R, Pasini E, Meletti $S$ et al. Epilepsy in primary cerebral tumors: the characteristics of epilepsy at the onset (results from the PERNO study - Project of Emilia Romagna Region on Neuro-Oncology). Epilepsia 54(Suppl. 7), S86-S91 (2013). 